\title{
Optimal Dispatch of Integrated Community Energy System Considering Energy Flexibilities in Pipelines and Buildings
}

\author{
Yan $\mathrm{Qi}^{1,2}$, Lei $\mathrm{Wu}^{1,2}$, Yi Ding ${ }^{1,2}$, Hairun $\mathrm{Li}^{3}$ \\ ${ }^{1}$ State Grid Tianjin Electric Power Research Institute, Tianjin, China \\ ${ }^{2}$ Tianjin Key Laboratory of Internet of Things in Electricity, Tianjin, China \\ ${ }^{3}$ Key Laboratory of Smart Grid of Ministry of Education Tianjin University, Tianjin, China
}

\begin{abstract}
The development of cogeneration, distributed generation, information and communication technology has facilitated the application of integrated community energy system (ICES). However, some ICESs may lack operational flexibility due to the strict constraints of community heating system (CHS), which reduce the energy efficiency of ICESs. To solve this problem, an optimal dispatch model of ICES considering energy flexibilities in pipelines and buildings is proposed, in which the thermal dynamics of pipelines and buildings are described by node method and equivalent thermal parameter (ETP) method. The objective of the proposed dispatch method is to minimize the daily operation cost of ICES. What's more, renewable energy accommodation cost and power purchase cost are both analysed to verify the improvement of ICES flexibility. It is demonstrated that the proposed method can unlock the flexibility of CHS and improve the energy efficiency of ICES.
\end{abstract}

\section{Introduction}

Integrated Community Energy System (ICES) is a common solution for multi-energy integration and joint operation on the consumer-side [1][2]. It is currently the key target of the applications of multi-energy technology, which has the potential of improving economic efficiency, system reliability and renewable energy accommodation [3].

However, due to the strict constraints of community heating system (CHS), ICES flexibility may be restricted. In the face of high penetration of renewable energy resources and fast-growing energy demand, the lack of flexibility can deteriorate into insecurity of energy supply.

Some studies have been done to access the flexibility of heating system and improve the performance of multienergy operation. Heating pipelines are considered to be short-term energy storage to improve the economic efficiency of district heating system in [4], and an annual evaluation model is established. In [5], a quantification method for the thermal energy storage flexibility of building energy system is proposed. And an optimal scheduling framework of integrated power and heat system considering thermal inertia of pipelines and buildings is proposed in [6], which can improve wind accommodation. In addition to thermal storage devices, pipelines and buildings are the two main focuses of flexibilities in CHS. For simplification, these two types of flexibilities are denoted as CHS flexibility in the following paragraphs.
This paper proposes an optimal dispatch model of ICES considering flexibilities in pipelines and buildings. In the following section, the flexibility of CHS is formulated, and the optimal dispatch model is denoted as a LP problem. Then, the effectiveness of the proposed method is verified by the case study.

\section{Configuration of ICES}

The configuration of ICES is shown in Fig. 1, which is composed of energy inputs, energy conversion devices and community loads. The energy input takes into account the power purchase from power grid and the natural gas from gas supply pipeline. The energy conversion devices of ICES include wind turbine (WT), photovoltaic (PV), CHP unit, gas boiler (GB), electric boiler (EB) and power transformer. Community loads consist of electric load and heating load, in which the heating load is described by a detailed model of CHS.

The energy conversion process is modelled as an energy hub. The power load is supplied by the power grid, CHP units, wind turbine and photovoltaic; the heat load is supplied by CHP units, gas boilers and electric boilers. In the daily operation, ICES operator provides power supply and heating services to meet community consumers' electric and heating demands.

\footnotetext{
${ }^{\text {*} C o r r e s p o n d i n g ~ a u t h o r: ~ h a i r u n l i @ t j u . e d u . c n ~}$
} 


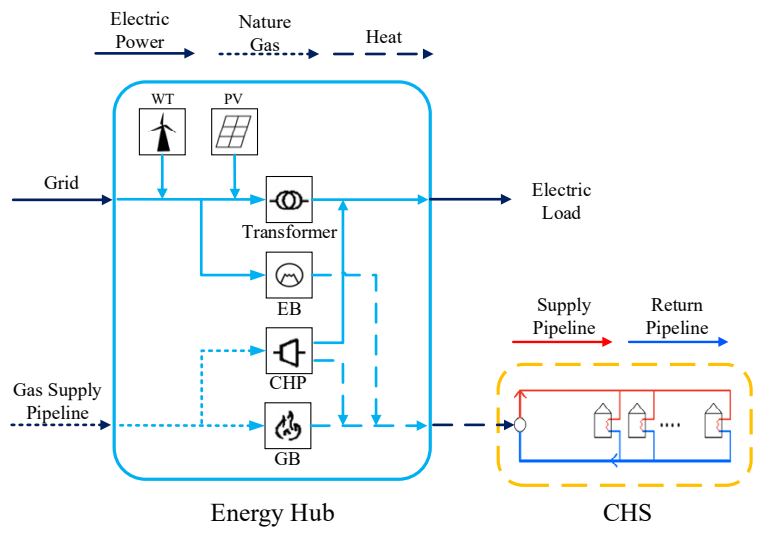

Fig. 1. Configuration of ICES

\section{Optimal dispatch model of ICES with CHS flexibilities}

\subsection{CHS flexibility formulation}

Traditional dispatch method assumes that the heating system operates in a "heat-led" mode, in which the heat power and heat load need to be balanced at each dispatch period. However, this assumption ignores the potential of flexibility in the thermal dynamics of pipelines and buildings. Pipelines with transmission delay can be regarded as an energy buffer between the heat source and the heat load, which means that the heat power and the heat load do not need to be balanced in real time. In addition, the indoor temperature can fluctuate slightly within the set interval without violating the thermal comfort of consumers, which further improves the flexible control ability of the heat source. In this subsection, node method and ETP method are applied to demonstrate the energy flexibility of pipelines and buildings.

\subsubsection{Flexibility formulation of pipelines}

In this paper, the widely used node method is adopted to describe the thermal dynamics in the heat transfer process of CHS pipelines.

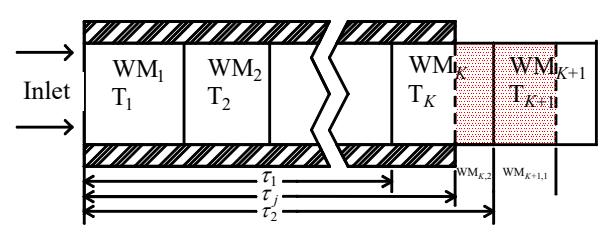

Fig. 2. Schematic diagram of transmission delay

As shown in Fig. 2, water is divided into a sequence of water masses along the pipeline. In this method, the outlet temperature is estimated with the inlet temperature in previous period, as follow:

$$
\begin{aligned}
T_{\tau, j}^{\text {out }}= & C_{1} C_{3} T_{\tau-\tau_{1}, j}^{\text {in }}+C_{2} C_{4} T_{\tau-\tau_{2}, j}^{\text {in }}+ \\
& \left(1-C_{1} C_{3}-C_{2} C_{4}\right) T^{\text {am }}
\end{aligned}
$$

$$
\begin{gathered}
\tau_{1}=\left\lceil\frac{l_{j}}{v_{\mathrm{w}} \Delta t}\right\rceil-1 \\
\tau_{2}=\left\lceil\frac{l_{j}}{v_{\mathrm{w}} \Delta t}\right\rceil
\end{gathered}
$$

where $T_{\tau, j}^{\text {out }}$ and $T_{\tau, j}^{\text {in }}$ are the outlet and inlet temperature of pipeline $j$ at period $\tau ; T^{\mathrm{am}}$ is the ambient temperature; $l_{j}$ is the length of pipeline $j ; v_{\mathrm{w}}$ is the flow rate of water; $\Delta t$ is the dispatch interval; coefficients $C_{1}, C_{2}, C_{3}$ and $C_{4}$ represent transmmision delay and heat loss, and the detailed formulation of these coefficients can refer to our previous work [7].

At each node, the node temperature mixing equation describes the mixing law of the water temperature of the inflow node and the outflow node. The inflow water with different temperature is uniformly mixed, as below:

$$
\sum_{j} T_{\tau, j}^{\text {out }} m_{\tau, j}=T_{\tau, k}^{\text {in }} \sum_{k} m_{\tau, k}
$$

where $m_{\tau, j}$ is the outflow mass flow rate of pipeline $j$ at period $\tau ; m_{\tau, k}$ is the inflow mass flow rate of pipeline $k$ at period $\tau$. The primary heat exchange station is formulated as:

$$
Q_{\tau}^{\text {source }}=c_{\mathrm{w}} m_{\tau}^{\text {source }}\left(T_{\tau}^{\text {supply }}-T_{\tau}^{\text {return }}\right)
$$

where $Q_{\tau}^{\text {source }}$ is the thermal power of the heat source at period $\tau ; c_{\mathrm{w}}$ is the specific heat capacity of water; $m_{\tau}^{\text {source }}$ is the mass flow rate at the primary heat exchange station at period $\tau ; T_{\tau}^{\text {supply }}$ and $T_{\tau}^{\text {return }}$ are the supply and return temperature at the primary heat exchange station at period $\tau$.

\subsubsection{Flexibility formulation of buildings}

In this paper, we assume that all heat loads connected to the heating network are space heating. Thus, each node of heat load is considered as an equivalent building. The energy balance equation of heat load is stated as bellow:

$$
H_{\tau, i}^{\text {load }}=c_{\mathrm{w}} m_{\tau, i}\left(T_{\tau, i}^{\text {supply }}-T_{\tau, i}^{\text {return }}\right)
$$

where $H_{\tau, i}^{\text {load }}$ is the thermal power of heat load $i$ at period $\tau ; m_{\tau, i}$ is the mass flow rate of heat load $i$ at period $\tau ; T_{\tau, i}^{\text {supply }}$ and $T_{\tau, i}^{\text {return }}$ are the supply and return temperature of heat load $i$ at period $\tau$.

Besides, ETP method is applied to describe the temperature variation in equivalent building:

$$
H_{\tau, i}^{\text {load }}=C_{i}^{\text {load }}\left(T_{\tau, i}^{\text {load }}-T_{\tau-1, i}^{\text {load }}\right) / \Delta t+\left(T_{\tau, i}^{\text {load }}-T^{\text {am }}\right) / R_{i}^{\text {load }}
$$

where $T_{\tau, i}^{\text {load }}$ is the average indoor temperature of load $i$ at period $\tau ; C_{i}^{\text {load }}$ is the thermal capacity of load $i$; $R_{i}^{\text {load }}$ is the thermal resistance of load $i$. 


\subsection{Optimal dispatch of ICES}

\subsubsection{Objective function}

The main goal of the ICES optimal dispatch model with CHS flexibilities is to minimize the operational cost of the ICES while satisfying the electrical and thermal demand of community consumers. The objective function of the proposed dispatch model is denoted as $C$, which includes the cost of purchasing electricity from the upper-level grid, the cost of purchasing gas from the upper-level gas supply grid, and the cost for renewable energy curtailment. The expression of $C$ and its components are stated as:

$$
\begin{aligned}
& C=C_{\mathrm{E}}+C_{G}+C_{\mathrm{WT}, \text { loss }}+C_{\mathrm{PV}, \text { loss }} \\
& \left\{\begin{array}{l}
C_{\mathrm{E}}=\sum_{\tau=1}^{N} k_{\tau}^{\mathrm{E}} P_{\tau}^{\mathrm{in}} \Delta t \\
C_{\mathrm{G}}=\sum_{\tau=1}^{N} k_{\tau}^{\mathrm{G}} G_{\tau}^{\mathrm{in}} \Delta t \\
C_{\mathrm{WT}, \text { loss }}=\sum_{\tau=1}^{N} k_{\tau}^{\mathrm{WT}, \text { loss }} P_{\tau}^{\mathrm{WT}, \text { loss }} \Delta t \\
C_{\mathrm{PV}, \text { loss }}=\sum_{\tau=1}^{N} k_{\tau}^{\mathrm{pV}, \text { loss }} P_{\tau}^{\mathrm{PV}, \text { loss }} \Delta t
\end{array}\right.
\end{aligned}
$$

where $C_{\mathrm{E}}, C_{\mathrm{G}}, C_{\mathrm{WT} \text {,loss }}$ and $C_{\mathrm{PV} \text {,loss }}$ are the cost of power purchase, gas purchase, wind curtailment and photovoltaic curtailment; $k_{\tau}^{E}$ is the price of electricity brought from the main grid; $k_{\tau}^{G}$ is the price of natural gas; $P_{\tau}^{\text {in }}$ is the power brought from main grid; $G_{\tau}^{\text {in }}$ is the inflow natural gas; $k_{\tau}^{\mathrm{wT} \text {,loss }}$ and $k_{\tau}^{\mathrm{PV} \text { loss }}$ are unit-price of curtailed wind and photovoltaic; $P_{\tau}^{\mathrm{WT} \text {,loss }}$ and $P_{\tau}^{\mathrm{PV} \text {,loss }}$ are curtailed wind and photovoltaic at period $\tau$.

\subsection{Constraints}

1)constraints for power balance

In order to maintain the safety and efficiency of ICES, constraints for power balance in electricity, heat and gas is needed:

$$
\begin{gathered}
P_{\tau}^{\mathrm{in}}+P_{\tau}^{\mathrm{CHP}}+P_{\tau}^{\mathrm{WT}}+P_{\tau}^{\mathrm{PV}}=P_{\tau}^{\mathrm{load}}+P_{\tau}^{\mathrm{EB}} \\
Q_{\tau}^{\mathrm{CHP}}+Q_{\tau}^{\mathrm{GB}}+Q_{\tau}^{\mathrm{EB}}=Q_{s, \tau} \\
G_{\tau}^{\mathrm{CHP}}+G_{\tau}^{\mathrm{GB}}=G_{\tau}^{\mathrm{in}}
\end{gathered}
$$

Where $P_{\tau}^{\mathrm{CHP}}, P_{\tau}^{\mathrm{WT}}, P_{\tau}^{\mathrm{PV}}, P_{\tau}^{\text {load }}$ and $P_{\tau}^{\mathrm{EB}}$ are the electric power of CHP, wind turbine, photovoltaic, electric load and electric boiler; $Q_{\tau}^{\mathrm{CHP}}, Q_{\tau}^{\mathrm{GB}}, Q_{\tau}^{\mathrm{EB}}$ and $Q_{\tau}^{\text {source }}$ are the heat power of CHP, gas boiler, electric boiler and heat source; $G_{\tau}^{\mathrm{CHP}}$ and $G_{\tau}^{\mathrm{GB}}$ are the gas power of CHP and gas boiler.

When CHS operate under "heat-led" mode, another constraint of heat power balance should be applied, which is stated as:

$$
Q_{\tau}^{\text {source }}=\sum_{i} Q_{\tau, i}^{\text {load }}
$$

2)constraints for energy devices

Energy devices constraints consist of energy conversion constraints, output upper and lower limit constraints, and ramp rate constraints.

In this paper, a back pressure CHP with fixed powerto-heat ratio is used. The energy conversion constraint of CHP reflects the relationship between CHP gas consumption, heat generation, and power generation:

$$
\begin{gathered}
Q_{\tau}^{\mathrm{CHP}}=\varepsilon P_{\tau}^{\mathrm{CHP}} \\
Q_{\tau}^{\mathrm{CHP}}=\eta G_{\tau}^{\mathrm{CHP}} \\
\eta=\frac{\varepsilon \eta_{\mathrm{CHP}} b_{\mathrm{v}}}{\varepsilon+1}
\end{gathered}
$$

where $\varepsilon$ is power-to-heat ratio; $\eta$ is gas-heat conversion efficiency; $\eta_{\mathrm{CHP}}$ is CHP efficiency; $b_{\mathrm{v}}$ is the combustion calorific value of natural gas. Constraints of CHP output and ramp rate are stated as:

$$
\begin{gathered}
P_{\min }^{\mathrm{CHP}} \leq P_{\tau}^{\mathrm{CHP}} \leq P_{\max }^{\mathrm{CHP}} \\
-S_{\mathrm{DR}}^{\mathrm{CHP}} \Delta t \leq P_{\tau+1}^{\mathrm{CHP}}-P_{\tau}^{\mathrm{CHP}} \leq S_{\mathrm{UR}}^{\mathrm{CHP}} \Delta t
\end{gathered}
$$

where $P_{\max }^{\mathrm{CHP}}$ and $P_{\min }^{\mathrm{CHP}}$ are output limits of CHP; $S_{\mathrm{UR}}^{\mathrm{CHP}}$ and $S_{\mathrm{DR}}^{\mathrm{CH}}$ are ramp limits of CHP.

For gas boiler, the constraints of energy conversion, output and ramp rate are stated as:

$$
\begin{gathered}
Q_{\tau}^{\mathrm{GB}}=\eta_{\mathrm{GB}} G_{\tau}^{\mathrm{GB}} L_{\mathrm{HANG}} \\
Q_{\mathrm{min}}^{\mathrm{GB}} \leq Q_{\tau}^{\mathrm{GB}} \leq Q_{\mathrm{max}}^{\mathrm{GB}} \\
-S_{\mathrm{DR}}^{\mathrm{GB}} \leq Q_{\tau+1}^{\mathrm{GB}}-Q_{\tau}^{\mathrm{GB}} \leq S_{\mathrm{UR}}^{\mathrm{GB}}
\end{gathered}
$$

where $\eta_{\mathrm{GB}}$ is the gas-heat conversion efficiency of gas boiler; $L_{\mathrm{HANG}}$ is the low calorific value of natural gas; $Q_{\max }^{\mathrm{GB}}$ and $Q_{\min }^{\mathrm{GB}}$ are output limits of gas boiler; $S_{\mathrm{UR}}^{\mathrm{GB}}$ and $S_{\mathrm{DR}}^{\mathrm{GB}}$ are ramp limits of gas boiler.

For electric boiler, the formulation of the constraints of energy conversion, output and ramp rate are similar:

$$
\begin{gathered}
Q_{\tau}^{\mathrm{EB}}=\eta_{\mathrm{EB}} P_{\tau}^{\mathrm{EB}} \\
Q_{\mathrm{min}}^{\mathrm{EB}} \leq Q_{\tau}^{\mathrm{EB}} \leq Q_{\max }^{\mathrm{EB}} \\
-S_{\mathrm{DR}}^{\mathrm{EB}} \leq Q_{\tau+1}^{\mathrm{EB}}-Q_{\tau}^{\mathrm{EB}} \leq S_{\mathrm{UR}}^{\mathrm{EB}}
\end{gathered}
$$

where $\eta_{\mathrm{EB}}$ is the power-heat conversion efficiency of electric boiler; $Q_{\max }^{\mathrm{EB}}$ and $Q_{\min }^{\mathrm{EB}}$ are output limits of electric boiler; $S_{\mathrm{UR}}^{\mathrm{EB}}$ and $S_{\mathrm{DR}}^{\mathrm{EB}}$ are ramp limits of electric boiler.

When ICES lacks flexibility, wind and photovoltaic outputs are constrained. The constraints of renewable energy output are states as:

$$
\begin{gathered}
0 \leq P_{\tau}^{\mathrm{WT}} \leq P_{\tau}^{\mathrm{WT}, \text { curve }} \\
P_{\tau}^{\mathrm{WT}}+P_{\tau}^{\mathrm{WT}, \text { loss }}=P_{\tau}^{\mathrm{WT}, \text { curve }} \\
0 \leq P_{\tau}^{\mathrm{PV}} \leq P_{\tau}^{\mathrm{PV}, \text { curve }}
\end{gathered}
$$




$$
P_{\tau}^{\mathrm{PV}}+P_{\tau}^{\mathrm{PV}, \text { loss }}=P_{\tau}^{\mathrm{PV}, \text { curve }}
$$

where $P_{\tau}^{\mathrm{WT} \text {,curve }}$ and $P_{\tau}^{\mathrm{PV} \text {,curve }}$ are the forecasted output of wind turbine and photovoltaic.

3)constraints for $\mathrm{CHS}$

Besides (1)-(7), each temperature variable is restricted by its bound, including constraints for supply and return temperature as well as indoor temperature. These constraints can be stated as:

$$
\begin{gathered}
T_{\min }^{\text {supply }} \leq T_{\tau, j}^{\text {supply }} \leq T_{\max }^{\text {supply }} \\
T_{\min }^{\text {return }} \leq T_{\tau, j}^{\text {return }} \leq T_{\max }^{\text {return }} \\
T_{\min }^{\text {load }} \leq T_{\tau, i}^{\text {load }} \leq T_{\max }^{\text {load }}
\end{gathered}
$$

\section{Case study}

A typical ICES is adopted as shown in Fig. 1. The EH model of ICES includes CHP, gas boiler, electric boiler, power transformer, wind turbine, and photovoltaic. The maximum electric output of CHP unit is $12 \mathrm{MW}$. And the capacity of electric boiler and gas boiler are $1.5 \mathrm{MW}$ and 2 MW. For CHS, we have adopted a 44-node heating network [7].

This article selects the dispatch period as 24 hours, and the scheduling period is 1 hour. The predicted output curves of ICES system electrical load, thermal load, wind turbine and photovoltaic are shown in Fig. 3. The electricity price and the forecasted value of the temperature of the dispatch day are shown in Fig. 4. The price of natural gas is $1.72 ¥ / \mathrm{kWh}$, and the penalty cost for wind and photovoltaic curtailment is $0.1 ¥ / \mathrm{kWh}$. The temperature limits of the water supply and return are $60^{\circ} \mathrm{C}-90^{\circ} \mathrm{C}$ and $20^{\circ} \mathrm{C}-40^{\circ} \mathrm{C}$, respectively. And the indoor temperature limit is set between $18^{\circ} \mathrm{C}$ and $24^{\circ} \mathrm{C}$.

The proposed optimal dispatch method is formulated as LP problem which can be solved by general optimization solver.

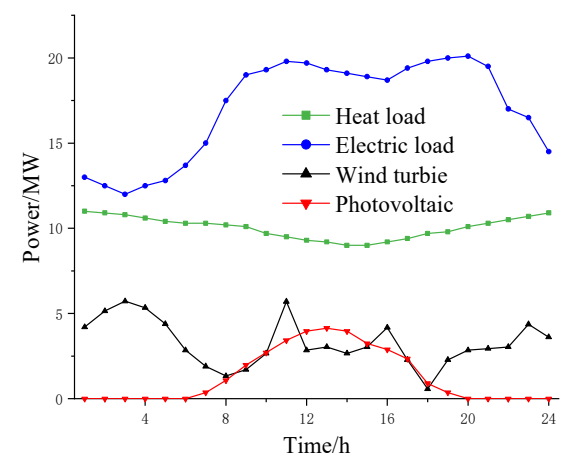

Fig. 3. ICES load and available wind and solar power

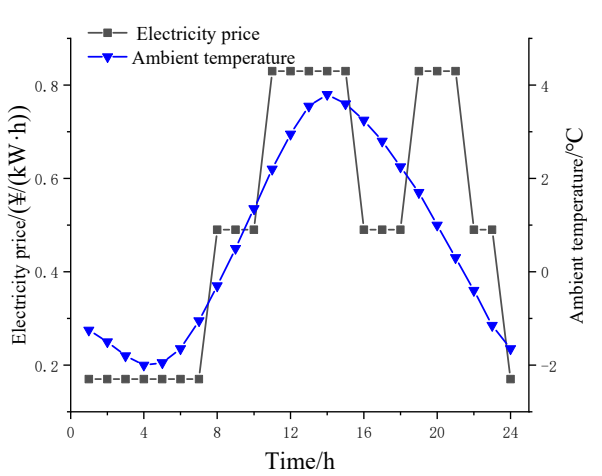

Fig. 4. Electricity price and ambient temperature

Two case are set to demonstrate the effectiveness of the proposed method.

Case I: The proposed optimal dispatch model with CHS flexibility.

Case II: ICES dispatch model with CHS operating under traditional "heat-led" mode.

Firstly, operational cost of two cases is compared in Table 1. Although curtailment occurs in both cases, the curtailment cost in case I is $65 \%$ lower than that in case II. And the electricity purchase cost in case I is $42 \%$ lower than that in case II. In total, case I has saved 9\% of total cost compared with case II.

Table 1. Operational cost of two cases

\begin{tabular}{|c|c|c|c|c|}
\hline Case & $C / ¥$ & $C_{\mathrm{E}} / ¥$ & $C_{\mathrm{G}} / ¥$ & $\begin{array}{c}C_{\mathrm{WT}, \text { loss }}+C_{\mathrm{PV}, \text { loss }} / \\
¥\end{array}$ \\
\hline Case I & 114902 & 13606 & 100559 & 737 \\
\hline Case II & $\mathbf{1 2 6 2 2 5}$ & 23492 & 100634 & 2129 \\
\hline
\end{tabular}

Then, power and heat balance of two cases is compared as shown in Fig. 5 and Fig. 6. The renewable energy curtailment reduces significantly in case I, in which CHP output becomes more adjustable in case I. From Fig. 6 we can see heat power equals to heat load in case II under "heat-led" mode. In contrast, heat power and heat load do not balance in real time necessarily in the proposed model.

Moreover, we can conclude from Fig. 5 (b) that CHP plays an important role in improving ICES flexibility under two types of motivation. On the one hand, CHP tends to decrease power output in wind and photovoltaic curtailment hours. On the other hand, CHP generates more power when electricity price reaches peak level.

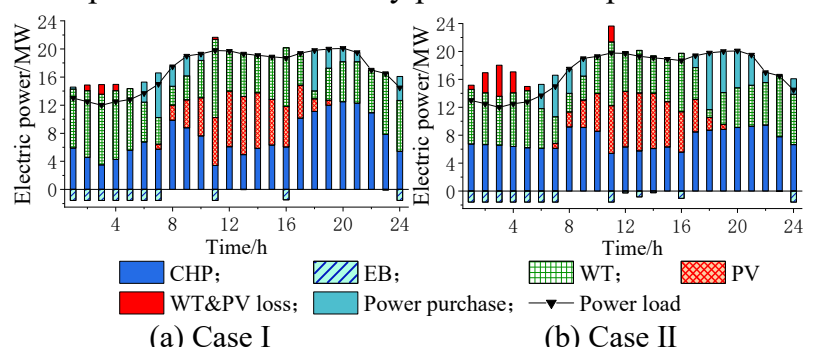
(a) Case I
(b) Case I

Fig. 5. Electricity power balance of ICES 


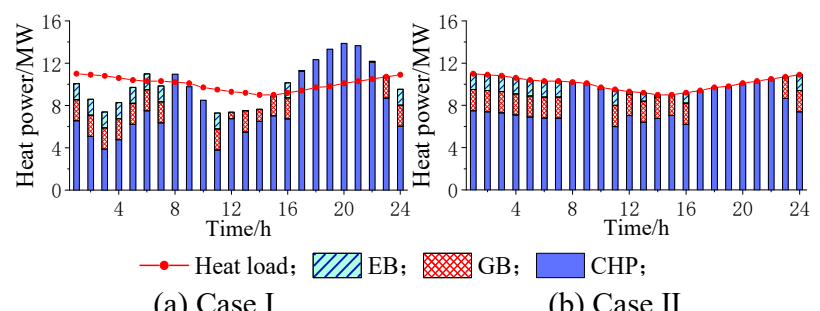

(a) Case I

(b) Case II

Fig. 6. Heat power balance of ICES

In order to investigate the source of CHS flexibility, return temperature and heat power imbalance of CHS in case II are shown in Fig. 6. The imbalanced heat power between power and load represents the charging and discharging process of thermal energy in CHS. The proposed dispatch model considers existing facilities as extra energy storage devices in the ICES operation, thus unlocking the flexibility of CHS. Besides, return temperature also denotes the approximate energy storage level of CHS.

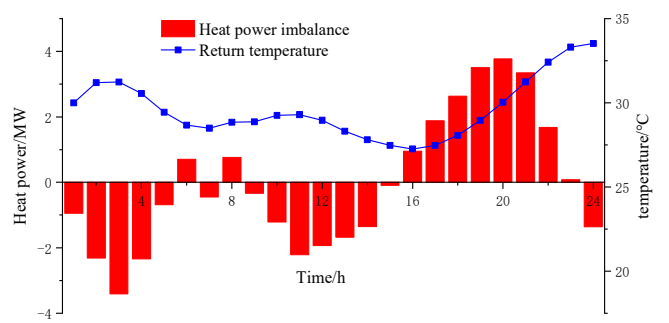

Fig. 7. Return temperature and heat power imbalance of CHS

\section{Conclusions}

This paper proposes an optimal dispatch model for ICES which exploits the flexibility of CHS. In the flexibility formulation of CHS, both flexibilities of pipelines and buildings are considered using node method and ETP method. The results of case study show that the proposed dispatch method can unlock the flexibility of CHS, and improve the economic efficiency, renewable energy accommodation and flexibility of ICES operation.

\section{Acknowledgements}

This work was financially funded by the Science and Technology Project of State Grid Tianjin Electric Power Company (KJ20-1-29).

\section{References}

1. H. Jia, Y. Mu, X. Yu. Thought about the integrated energy system in China. Electric Power Construction 36(01), 16-25 (2015)

2. K. Peng, C. Zhang, B. Xu, et al. Status and prospect of demonstration project of multi-energy synergy integrated energy system. Electric Power Automation Equipment 37(06), 3-10 (2017)
3. D. Wang, L. Liu, H. Jia, et al. Review of key problems related to integrated energy distribution systems. CSEE Journal of Power and Energy Systems 4(2), 130-145 (2018)

4. M. Kouhia, T. Laukkanen, H. Holmberg, et al. District heat network as a short-term energy storage. Energy 177, 293-303 (2019)

5. S. Stinner, K. Huchtemann, D. Müller. Quantifying the operational flexibility of building energy systems with thermal energy storages. Applied Energy 181, 140-154 (2016)

6. W. Gu, J. Wang, S. Lu, et al. Optimal operation for integrated energy system considering thermal inertia of district heating network and buildings. Applied Energy 199, 234-246 (2017)

7. M. Wang, Y. Mu, X. Meng, et al. Optimal Scheduling Method for Integrated Electro-thermal Energy System Considering Heat. Power System Technology 44, 132-140 (2020) 